\title{
Large-Volume Paracentesis in Patients with Cirrhotic Ascites: Does It Increase the Risk of Serious Bleeding and the Need for Transfusion?
}

\author{
Hind I. Fallatah \\ Unit of Hepatology and Gastroenterology, Department of Internal Medicine, Faculty of Medicine, King \\ Abdulaziz University, Jeddah, Saudi Arabia \\ Email: hindfallatah@hotmail.com
}

Received 19 November 2015; accepted 22 December 2015; published 25 December 2015

Copyright (C) 2015 by author and Scientific Research Publishing Inc.

This work is licensed under the Creative Commons Attribution International License (CC BY). http://creativecommons.org/licenses/by/4.0/

(c) (i) Open Access

\begin{abstract}
Background: Liver cirrhosis is the most common cause of ascites. For cirrhotic ascites that does not respond to diuretics and salt restriction, large-volume paracentesis is an alternative option. Methods: A retrospective cohort study of patients admitted to the Day care unit at King Abdulaziz University Hospital for therapeutic paracentesis of cirrhotic ascites was performed from March 2013-April 2014. The demographic data and results, including the platelet count, hemoglobin level, prothrombin time (PT), international normalized ratio (INR), serum creatinine, serum albumin, and bilirubin levels, were recorded. We recorded all of the bleeding episodes. Results: We recorded 118 admissions for 13 patients. Nine of them were male (69.2\%), and the mean age was $58.6 \pm 15.8$ years. All patients had a Child-Pugh score of $C$. The platelet count was lower than normal for 78 admissions (66.1\%), and the PT was prolonged for 99 admissions (84\%). Three episodes of bleeding occurred in our cohort, all of which were mild and controlled by the local application of pressure. One patient required a platelet transfusion for severe thrombocytopenia, low platelets count was associated with elevated creatinine and low albumin levels $(P=0.014$ and 0.003 , respectively). Similarly, a prolonged PT was associated with low albumin, high bilirubin, low platelet, and high creatinine levels $(P=0.013,<0.001,=0.006$, and $<0.001$, respectively $)$. Conclusions: Large-volume paracentesis is associated with only a small risk of bleeding in patients with cirrhotic ascites, and a transfusion of fresh frozen plasma (FFP) and platelets is not needed for the majority of patients.
\end{abstract}

\section{Keywords}

Cirrhosis, Ascites, Paracentesis, Ultrasound, Prolonged PT 


\section{Introduction}

Liver cirrhosis is the most common cause of ascites [1]. The standard treatment for cirrhotic ascites is salt restriction and diuresis with spironolactone and furosemide [2] [3]. The diuretic dose is generally adjusted until an optimal response is achieved [2] [3]. However, many patients with cirrhotic ascites fail to respond to the maximum dose of diuretics and are classified as having refractory ascites [3]-[5]. In contrast, failure to optimize the dose of diuretics due to the side effects of the treatment is defined as diuretic intractable ascites [2]-[5]. The management of diuretic refractory and intractable cirrhotic ascites includes frequent therapeutic paracentesis (removing more than five liters of ascetic fluid), which can be continued until liver transplantation or the insertion of a transjugular portosystemic shunt [2]-[5]. However, most patients with advanced liver cirrhosis have coagulation disorders due to hepatic dysfunction, platelet dysfunction and/or thrombocytopenia due to hypersplenism [6]-[8]. These factors are thought to place cirrhotic patients with therapeutic paracentesis, especially those with advanced cirrhosis at risk of bleeding [6] [7]. At King Abdulaziz University Hospital, we have frequently discussed the need for fresh frozen plasma transfusions for cirrhotic patients undergoing ultrasound-guided therapeutic paracentesis with our radiologists. This study was conducted to determine whether abdominal paracentesis was related to bleeding episodes in patients with refractory cirrhotic ascites who were admitted to the day care unit for therapeutic paracentesis.

\section{Material and Methods}

This was a retrospective cohort study conducted from March 2013 to April 2014. Ethics committee approval was obtained for this study.

The inclusion criteria consisted of patients with liver cirrhosis who were under the care of the hepatology department, had confirmed diuretic-refractory or intractable cirrhotic ascites and had been admitted to the day care unit at King Abdulaziz University Hospital Jeddah for therapeutic paracentesis (defined as the aspiration of five or more liters of ascetic fluid). For each liter of ascetic fluid removed, $10 \mathrm{~g}$ of intravenous human albumin was administered.

We obtained the demographic data for each patient, including the age, sex, cause of liver disease and all reported admissions during the study period. For each admission episode, we obtained data for the following parameters: hemoglobin level, platelet count, serum creatinine level, serum albumin level, prothrombin time (PT) and international normalized ratio (INR) (see Table 1 for normal ranges). Paracentesis was performed in all patients under ultrasound guidance. We recorded all of the bleeding episodes that developed during paracentesis.

SPSS 22 was used for the statistical analyses. Descriptive statistics were obtained, and we performed a correlation analysis to identify factors associated with low platelet counts and a multiple regression analysis to study the relationship of prolonged PT with other factors.

\section{Results}

The study included 118 admissions of 13 patients, nine males and four females. The mean age was $58.6 \pm 15.8$ years. Hepatitis $C$ was the most common cause of cirrhosis (present in four patients), followed by NAFLD and AIH (three patients each), and then Hepatitis B, bilharzia liver disease and hepatocellular carcinoma complicating hepatitis $\mathrm{C}$ (one patient each). All patients had advanced cirrhosis and were categorized as Child-Pugh class C. Three episodes (2.42\%) of non-life-threatening bleeding occurred, all of which were controlled by the application of pressure to the bleeding area and a blood transfusion. None of the episodes required hospital admission or consultation with a major inpatient department. For one episode, the procedure was postponed due to bleeding and because the platelet count was 9. The patient underwent the rescheduled paracentesis after receiving a platelet transfusion. The mean serum albumin level, platelet count and hemoglobin level were low, and the mean serum PT and INR values were above the normal range (see Table 1).

For 99 (84\%) of the admissions, the PT was higher than normal (>4). Similarly, for 78 admissions (66.1\%), the platelet count was below normal $(<150)$, and it was below 100 in 54 admissions $(45.8 \%)$. Moreover, the platelet count showed a significant inverse correlation with the serum creatinine and serum albumin levels (Pearson $r=265, \mathrm{P}=0.014$ and Pearson $\mathrm{r}=0.33, \mathrm{P}=0.003$, respectively). In the multiple regression analysis, the following factors were found to be associated with a prolonged PT: thrombocytopenia, elevated serum creatinine level, elevated bilirubin level and low albumin level (Table 2). None of the patients exhibited any major complications that required admission to a major care department. 
Table 1. Results of the laboratory tests for all patients and the normal ranges.

\begin{tabular}{ccccc}
\hline Lab test, normal range & Minimum & Maximum & Mean & Standard deviation \\
\hline HG, 12 - $15 \mathrm{~g} / \mathrm{dL}$ & 7 & 12 & 9.18 & 0.905 \\
Platelet count, 150 - $450 \mathrm{~K} / \mu \mathrm{L}$ & 9 & 294 & 106.8 & 48.23 \\
ALT, 30 - $65 \mathrm{U} / \mathrm{L}$ & 10 & 75 & 19.32 & 9.239 \\
Total bilirubin, 0 - $17 \mu \mathrm{mol} / \mathrm{L}$ & 3 & 98 & 13.47 & 16.456 \\
PT, 11 - 14 seconds & 11 & 33 & 13.89 & 0.18 \\
INR & 1 & 2 & 1.2 & 5.125 \\
Albumin, $35-50 \mathrm{~g} / \mathrm{L}$ & 10 & 33 & 21.92 & 3.191 \\
Urea, $2.4-6.4 \mathrm{mmol} / \mathrm{L}$ & 3 & 17 & 8.00 & 39.675 \\
Creatinine, 53 - $115 \mu \mathrm{mol} / \mathrm{L}$ & 54 & 323 & 115.20 & \\
\hline
\end{tabular}

"PT: prothrombin time. ${ }^{* *}$ INR: international normalized ratio.

Table 2. The results of the multiple regression analysis of factors associated with a prolonged prothrombin time.

\begin{tabular}{|c|c|c|c|c|c|c|}
\hline & \multirow{2}{*}{ Model } & \multicolumn{2}{|c|}{ Non-standardized coefficients } & \multirow{2}{*}{$\begin{array}{c}\text { Standardized coefficients } \\
\text { Beta }\end{array}$} & \multirow{2}{*}{$\mathrm{t}$} & \multirow{2}{*}{$P$ value } \\
\hline & & B & Std. Error & & & \\
\hline \multirow{7}{*}{1} & (Constant) & 14.042 & 1.941 & & 7.234 & 0.000 \\
\hline & $\mathrm{Hg}$ & -0.056 & 0.234 & -0.017 & -0.238 & 0.813 \\
\hline & Bilirubin & 0.127 & 0.014 & 0.680 & 9.369 & 0.000 \\
\hline & Albumin & -0.129 & 0.050 & -0.200 & -2.565 & 0.013 \\
\hline & Urea & -0.145 & 0.108 & -0.136 & -1.343 & 0.185 \\
\hline & Creatinine & 0.040 & 0.010 & 0.423 & 3.777 & 0.000 \\
\hline & Platelets & -0.015 & 0.005 & -0.220 & -2.847 & 0.006 \\
\hline
\end{tabular}

a. Dependent Variable: PT.

\section{Discussion}

The major finding of this study is that in patients with advanced cirrhosis and severe coagulopathy, therapeutic paracentesis did not lead to a significant bleeding risk. In the majority of admission episodes, patients had a prolonged PT, placing them at possible risk of bleeding. This study shows that fresh frozen plasma (FFP) transfusion can be safely deferred in these patients. Our present findings are consistent with those shown by Lin et al. [9]. The rate of bleeding in our cohort was low, with a rate similar to those shown by previous investigators [9]-[11]. These findings are relevant for avoiding unnecessary FFP and platelet transfusions in patients with advanced liver disease, which will help to avoid transfusion-related complications and volume overload [6] [12]-[14].

Coagulopathy in patients with advanced cirrhosis is due to several factors, including low vitamin K and vitamin K-dependent factor levels, nutritional deficiencies, reduced synthesis of protein $\mathrm{C}$ and protein S, dysfibrinogenemia and enhanced fibrinolysis [8] [12] [14] [15]. Data regarding the transfusion of FFP, platelets and other hemostatic agents are limited, and unnecessary transfusions can sometimes induce thrombosis [8] [14] [15]. In addition, advanced liver cirrhosis is also associated with an increased risk of intravascular coagulopathy and thrombosis [8] [14] [15]. Significant hypotension can sometimes develop as a result of therapeutic paracentesis, but in our cohort, none of the patients developed significant hypotension related to the paracentesis or the bleeding episodes. This result was likely related to the infusion of human albumin into our patients to replace that lost volume during the removal of ascites [16] [17]. All patients in our cohort were discharged home in sta- 
ble condition after the procedure. These results are similar to the findings of several previous reports on the safety of large-volume paracentesis [16] [17]. The laboratory parameters of the cohort in this study reflect the advanced state of their liver disease and the need for liver transplantation [18]. In addition to the other benefits of avoiding an unnecessary transfusion, deferring transfusion during paracentesis may also prevent the development of autoantibodies that could complicate future liver transplantation [19] [20].

\section{Conclusion}

This report shows that therapeutic paracentesis for cirrhotic ascites is not associated with serious bleeding. This finding demonstrates that there is no need for routine transfusion of FFP and platelets before performing paracentesis for cirrhotic patients.

\section{Acknowledgements}

The author would like to thank Josephine N. Floralde, the research assistant in the Gastroenterology and Hepatology unit, for her support in this study. Day care unit at KAUH, is a unit were the patients admitted only for few hours for treatment during the day time and discharge on the same day

\section{Conflict of Interest}

The author has no conflicts of interest to declare.

\section{Funding Source}

None. The human albumin that was used in the study was part of the routine King Abdulaziz University Hospital guidelines for the treatment of refractory cirrhotic ascites with paracentesis.

\section{References}

[1] Runyon, B.A. (1994) Care of Patients with Ascites. The New England Journal of Medicine, 330, 337-342. http://dx.doi.org/10.1056/NEJM199402033300508

[2] Hou, W. and Sanyal, A.J. (2009) Ascites: Diagnosis and Management. Medical Clinics of North America, 93, 801-817. http://dx.doi.org/10.1016/j.mcna.2009.03.007

[3] Moore, K.P. and Aithal, G.P. (2006) Guidelines on the Management of Ascites in Cirrhosis. Gut, 55, vi1-vi12. http://dx.doi.org/10.1136/gut.2006.099580

[4] Singhal, S., Baikati, K.K., Jabbour, I.I. and Anand, S. (2012) Management of Refractory Ascites. American Journal of Therapeutics, 19, 121-132. http://dx.doi.org/10.1097/MJT.0b013e3181ff7a8b

[5] Senousy, B.E. and Draganov, P.V. (2009) Evaluation and Management of Patients with Refractory Ascites. World Journal of Gastroenterology, 15, 67-80. http://dx.doi.org/10.3748/wjg.15.67

[6] Blonski, W., Siropaides, T. and Reddy, K.R. (2007) Coagulopathy in Liver Disease. Current Treatment Options in Gastroenterology, 10, 464-473. http://dx.doi.org/10.1007/s11938-007-0046-7

[7] Caldwell, S.H. (2014) Management of Coagulopathy in Liver Disease. Gastroenterology and Hepatology, 10, $330-332$.

[8] Amarapurkar, R.D. and Amarapurkar, D.N. (2011) Management of Coagulopathy in Patients with Decompensated Liver Cirrhosis. International Journal of Hepatology, 2011, Article ID: 695470.

[9] Lin, C.H., Shih, F.Y., Ma, M.H., Chiang, W.C., Yang, C.W. and Ko, P.C. (2005) Should Bleeding Tendency Deter Abdominal Paracentesis? Digestive and Liver Disease, 37, 946-951. http://dx.doi.org/10.1016/j.dld.2005.07.009

[10] De Gottardi, A., Thévenot, T., Spahr, L., Morard, I., Bresson-Hadni, S., Torres, F., Giostra, E. and Hadengue, A. (2009) Risk of Complications after Abdominal Paracentesis in Cirrhotic Patients: A Prospective Study. Clinical Gastroenterology and Hepatology, 7, 906-909. http://dx.doi.org/10.1016/j.cgh.2009.05.004

[11] Wiese, S.S., Mortensen, C. and Bendtsen, F. (2011) Few Complications after Paracentesis in Patients with Cirrhosis and Refractory Ascites. Danish Medical Bulletin, 58, A4212.

[12] Mannucci, P.M. and Tripodi, A. (2013) Liver Disease, Coagulopathies and Transfusion Therapy. Blood Transfusion, 11, 32-36.

[13] Amitrano, L., Guardascione, M.A., Brancaccio, V. and Balzano, A. (2002) Coagulation Disorders in Liver Disease. Seminars in Liver Disease, 22, 83-96. http://dx.doi.org/10.1055/s-2002-23205 
[14] Lisman, T. and Porte, R.J. (2010) Rebalanced Hemostasis in Patients with Liver Disease: Evidence and Clinical Consequences. Blood, 116, 878-885. http://dx.doi.org/10.1182/blood-2010-02-261891

[15] Peck-Radosavljevic, M. (2007) Review Article: Coagulation Disorders in Chronic Liver Disease. Alimentary Pharmacology \& Therapeutics, 26, 21-28. http://dx.doi.org/10.1111/j.1365-2036.2007.03509.x

[16] Nasr, G., Hassan, A., Ahmed, S. and Serwah, A. (2010) Predictors of Large Volume Paracantesis Induced Circulatory Dysfunction in Patients with Massive Hepatic Ascites. Journal of Cardiovascular Disease Research, 1, 136-144. http://dx.doi.org/10.4103/0975-3583.70914

[17] Wang, S.S., Chen, C.C., Chao, Y., Wu, S.L., Lee, F.Y., Lin, H.C., Kong, C.W., Tsai, Y.T. and Lee, S.D. (1996) Sequential Hemodynamic Changes for Large Volume Paracentesis in Post-Hepatitic Cirrhotic Patients with Massive Ascites. Proceedings of the National Science Council, Republic of China, Part B, 20, 117-122.

[18] O’Leary, J.G., Lepe, R. and Davis, G.L. (2008) Indications for Liver Transplantation. Gastroenterology, 134, 1764-1766.

[19] Kochhar, P.K., Ghosh, P. and Kochhar, R.S. (2012) Effect of Blood Transfusion on Subsequent Organ Transplantation. In: Kochhar, P., Ed., Blood Transfusion in Clinical Practice, Chap. 15, InTech, Rikeka, Croatia. http://www.intechopen.com/books/blood-transfusion-in-clinicalpractice/effect-of-blood-transfusion-on-subsequent-org an-transplantation http://dx.doi.org/10.5772/1868

[20] Scornik, J.C. and Meier-Kriesche, H.U. (2011) Blood Transfusions in Organ Transplant Patients: Mechanisms of Sensitization and Implications for Prevention. American Journal of Transplantation, 11, 1785-1791. http://dx.doi.org/10.1111/j.1600-6143.2011.03705.x 